\title{
The Effect of Dietary Safflower Phospholipid and Soybean Phospholipid on Plasma and Liver Lipids in Rats Fed a Hypercholesterolemic Diet
}

\author{
Toshio Iwata, ${ }^{1}$ Seiko Hoshi, ${ }^{2}$ Fumiyuki Takehisa, ${ }^{3}$ \\ Kentarou Tsutsumi, ${ }^{1}$ Yuji Furukawa, ${ }^{2}$ \\ and Shuichi KIMURA ${ }^{2}$ \\ ${ }^{1}$ Department of Research and Development, The Rinoru Oil Mills Co., Ltd., \\ Minato-ku, Nagoya 455, Japan \\ ${ }^{2}$ Laboratory of Nutrition, Department of Food Chemistry, \\ Faculty of Agriculture, Tohoku University, Aoba-ku, Sendai 981, Japan \\ ${ }^{3}$ Department of Home Economics, Miyagi-gakuin Women's College, \\ Sendai 981, Japan
}

(Received April 9, 1992)

Summary The effect of dietary safflower phospholipid (Saf-PL) and soybean phospholipid (Soy-PL) on plasma, liver, and fecal lipids in rats fed a hypercholesterolemic diet was compared with that of triglyceride mixture (controls). Triglyceride mixture (SP-Oil) of safflower oil and palm oil $(8: 2)$ contained almost comparable amounts of linoleic acid to safflower phospholipid or soybean phospholipid. Concentration of total cholesterol in plasma of rats fed the Saf-PL and Soy-PL diets were significantly decreased in comparison with that of the SP-Oil diet. Similarly, both Saf-PL and Soy-PL induced a reduction in the concentration of liver cholesterol compared with SP-Oil; Saf-PL indicated the lowest value. Saf-PL only significantly increased the level of high density lipoprotein (HDL) cholesterol. The level of chylomicron plus very low density lipoprotein (VLDL) cholesterol was lower in rats fed the Saf-PL and Soy-PL diets than that of the SP-Oil diet. The activity of plasma lecithin-cholesterol acyltransferase (LCAT) was increased in rats fed Saf-PL and Soy-PL. Saf-PL and Soy-PL caused an enhanced excretion of fecal neutral steroids, but not acidic steroids compared with SP-Oil. These results suggest that, in addition to soybean phospholipid, safflower phospholipid suppresses the elevation of plasma and liver cholesterol and that this effect may be brought about by inhibiting the absorption of cholesterol in the small intestine.

Key Words safflower phospholipid, soybean phospholipid, lipoprotein, plasma cholesterol, liver cholesterol, lecithin-cholesterol acyltransferase, fecal steroids, rats 
The effect of dietary phospholipids on serum lipids and lipoproteins has been extensively studied in humans (1-3) and rats (4-9). However, the mechanisms of cholesterol-lowering action of dietary phospholipids have still not been clarified. In a previous paper, we reported that safflower phospholipid suppressed the elevation of plasma and liver cholesterol in rats fed a hypercholesterolemic diet (10). We also showed that there was a significant difference of food intake between safflower phospholipid and triglyceride mixture (safflower oil : palm oil =8:2). Therefore, in order to equalize the food intakes between safflower phospholipid and triglyceride mixture, the feeding procedure was altered from ad libitum to pair-feeding. In this study, we have studied plasma and liver lipids and excretion of neutral and acidic steroids into feces in rats fed safflower phospholipid or soybean phospholipid compared with a triglyceride mixture containing comparable amounts of linoleic acid, respectively.

\section{MATERIALS AND METHODS}

Animals and diets. Male Sprague-Dawley rats, specific pathogen-free, were obtained from Funabashi Farm Co. (Funabashi). Each rat was individually housed in an air-conditioned room $\left(21-25^{\circ} \mathrm{C}\right)$ with lights on from $08: 00$ to $20: 00 \mathrm{~h}$. Rats weighing approximately $140 \mathrm{~g}$ were fed a commercial nonpurified diet (Type F-2, Funabashi Farm Co., Funabashi) for at least a week before initiation of the experiments with purified diets. The composition of the basal diet $(\mathrm{g} / 100 \mathrm{~g}$ diet $)$ was casein, 25; cellulose powder, 8 (Oriental Yeast Co., Tokyo); mineral mixture, 6; sucrose, 5; vitamin mixture, 2; cholesterol, 0.5; cholic acid, 0.25; and corn starch (Nippon Cornstarch Co., Aichi) to 100. Vitamin and mineral mixtures (Oriental Yeast Co., Tokyo) were according to Harper (11). As dietary lipids, safflower phospholipid (Rinoru Oil Mills Co., Ltd., Tokyo), soybean phospholipid (Rinoru Oil Mills Co., Ltd., Tokyo), and triglyceride mixture (safflower oil : palm oil=8:2) were added to diets at the level of $6 \%$. A food-restricted pair-fed safflower phospholipid and soybean phospholipid groups of rats were given the corresponding diets in an amount equal to that consumed on the previous day by their respective paired-mate ad libitum-fed triglyceride mixture rats. Food intake was measured every day and weight gain was measured weekly.

Analytical procedures. Safflower phospholipid and soybean phospholipid were analyzed according to the method of Watanabe et al. (12) by thin-layer chromatography (TLC) and the phospholipid class is shown in Table 1. The fatty acid composition of phospholipid and triglyceride was determined by gas-liquid chromatographic method of Metcalfe and Schmitz (13) and is shown in Table 1.

After 28-day feeding, ail rats were fasted overnight, and blood was collected from the abdominal aorta under diethyl ether anesthesia. Plasma lipoprotein was isolated at $4^{\circ} \mathrm{C}$ from each plasma sample by sequential ultracentrifugation in an RPL42T fixed-angle rotor of Hitachi ultracentrifuge (Mode! 70P-72, Hitachi, Ltd., Tokyo) according to the method of Havel et al. (14). The density $(d)$ ranges of 
Table 1. Phospholipid class and fatty acid composition of dietary fats used in the experiments $(\%)$.

\begin{tabular}{lccc}
\hline Dietary fat & SP-Oil & Saf-PL & Soy-PL \\
\hline Phospholipid content $(\mathbf{P} \times 25)$ & & 76.6 & 79.7 \\
\hline Phospholipid class & & & \\
Phosphatidylcholine & & 22.4 & 25.9 \\
Phosphatidylethanolamine & 14.9 & 18.0 \\
Phosphatidylinositol & 21.7 & 15.2 \\
Phosphatidic acid & 10.9 & 9.3 \\
Lysophosphatidylcholine & 4.2 & 3.4 \\
Lysophosphatidylethanolamine & & 5.5 & 2.2 \\
Others & & 20.4 & 26.0 \\
\hline Fatty acid composition & & & \\
$14: 0$ & 0.3 & 0.2 & 0.1 \\
$16: 0$ & 12.2 & 22.9 & 19.5 \\
$18: 0$ & 2.5 & 5.7 & 4.0 \\
$18: 1$ & 19.4 & 7.6 & 8.2 \\
$18: 2$ & 64.7 & 62.3 & 60.3 \\
$18: 3$ & 0.7 & 0.4 & 7.3 \\
$20: 0$ & - & 0.3 & - \\
$22: 0$ & 0.2 & 0.6 & 0.5 \\
$24: 0$ & - & - & 0.2 \\
\hline
\end{tabular}

SP-Oil, safflower oil and palm oil $(8: 2)$; Saf-PL, safflower phospholipid; Soy-PL, soybean phospholipid.

chylomicron plus very low density lipoproteins (VLDL), low density lipoproteins (LDL) and high density lipoproteins (HDL) were $d<1.006(\mathrm{~g} / \mathrm{ml}), d<1.063$ and $d<1.21$, respectively, using $\mathrm{KBr}$ solution.

Total cholesterol levels in plasma and lipoprotein fraction, free cholesterol, triglyceride and phospholipid levels in plasma were determined as described previously (10). Liver lipids were extracted in chloroform : methanol $=2: 1$ (15). Total cholesterol, triglyceride and phospholipid in liver were determined as described previously (10).

Activity of lecithin-cholesterol acyltransferase (LCAT, EC 2.3.1.43) was determined in plasma using $\left[{ }^{3} \mathrm{H}\right]$ cholesterol as a substrate according to the method of Stokke and Norum (16).

Feces were quantitatively collected for 2 days prior to death and immediately frozen at $-20^{\circ} \mathrm{C}$. Freeze-dried feces ( $500 \mathrm{mg}$ dry wt.) were saponified with $20 \mathrm{ml}$ of $0.2 \mathrm{~N} \mathrm{NaOH}$ at $65^{\circ} \mathrm{C}$ for $1 \mathrm{~h}$. Neutral steroids were then extracted with $3 \times 5 \mathrm{ml}$ of $n$-hexane and subjected to gas-liquid chromatography. The internal standard used was $5 \alpha$-cholestane (Sigma Chemical Co., USA). After the extraction of neutral steroids, acidic steroids were extracted according to the method of Ide and Horii (17) and were determined enzymatically with the Enzabile 2 kit (Daiichi 
Pure Chemicals Co., Tokyo).

Statistical analysis. Statistical evaluation of data was carried out by analysis of variance (ANOVA) coupled with a Duncan's New Multiple Range Test for the classification of the means. The acceptable level of probability was set at $95 \%$.

\section{RESULTS}

\section{Growth parameters and liver weight}

As shown in Table 2, weight gains of the Saf-PL and Soy-PL groups were significantly lower than that of the SP-Oil group. Liver weight $(\mathrm{g} / 100 \mathrm{~g}$ body weight) of rats fed the phospholipid diets was lower than that of the SP-Oil diet; Saf-PL indicated the lowest value. The plasmic activities of glutamic-pyruvic transaminase (GPT) and glutamic-oxaloacetic transaminase (GOT) were significantly lower in rats fed the phospholipid diets, when compared to rats fed the SPOil diet (GPT, 25.1 $\pm 3.5,23.8 \pm 2.1$ and 52.1 \pm 10.8 ; GOT, 93.9 $\pm 2.9,113 \pm 7.1$ and $142 \pm 8.2$ karmen units for Saf-PL, Soy-PL and SP-Oil, respectively).

\section{Plasma lipids}

Plasma lipid concentrations of rats fed the various experimental diets are shown in Table 3. The concentrations of plasma total cholesterol in rats fed the Saf-PL and Soy-PL diets were significantly decreased in comparison with those of the SP-Oil diet. Plasma triglyceride concentrations in rats fed the Saf-PL and Soy-PL diets tended to be low compared with those of rats fed the SP-Oil diet. Plasma free cholesterol and phospholipid concentrations did not differ among the various groups, respectively.

\section{Lipoprotein cholesterol}

The concentration of total cholesterol in the different lipoprotein fractions is shown in Table 3. Saf-PL significantly increased the level of HDL cholesterol in comparison with Soy-PL and SP-Oil. The level of LDL cholesterol tended to be low in rats fed the Saf-PL and Soy-PL diets compared with that of rats fed the

Table 2. Weight gain and liver weight of rats fed the experimental diets for 4 weeks.

\begin{tabular}{cccc}
\hline Dietary fat & $\begin{array}{c}\text { Initial body } \\
\text { weight }(\mathrm{g})\end{array}$ & $\begin{array}{c}\text { Weight gain } \\
(\mathrm{g} / 4 \text { weeks })\end{array}$ & $\begin{array}{c}\text { Liver weight } \\
(\mathrm{g} / 100 \mathrm{~g} \text { body weight })\end{array}$ \\
\hline SP-Oil & $191 \pm 3^{*}$ & $173 \pm 5^{\mathrm{b}}$ & $5.90 \pm 0.14^{\mathrm{b}}$ \\
Saf-PL & $190 \pm 4$ & $152 \pm 3^{\mathrm{a}}$ & $4.70 \pm 0.17^{\mathrm{a}}$ \\
Soy-PL & $190 \pm 4$ & $157 \pm 3^{\mathrm{a}}$ & $5.18 \pm 0.22^{\mathrm{a}}$ \\
\hline
\end{tabular}

SP-Oil, safflower oil and palm oil $(8: 2)$; Saf-PL, safflower phospholipid; Soy-PL, soybean phospholipid. ${ }^{*}$ Mean \pm SE $(n=10)$. Means in the same column not sharing a common superscript letter are significantly different $(p<0.05)$. 
Table 3. Concentrations of various lipids and activity of lecithin-cholesterol acyltransferase (LCAT) in plasma of rats fed the experimental diets for 4 weeks.

\begin{tabular}{lccc}
\hline \multicolumn{1}{c}{ Dietary fat } & SP-Oil & Saf-PL & Soy-PL \\
\hline & \multicolumn{3}{c}{$\mathrm{mg} / \mathrm{dl}$} \\
Total cholesterol & $157 \pm 9.0^{\mathrm{b} *}$ & $133 \pm 4.6^{\mathrm{a}}$ & $136 \pm 6.2^{\mathrm{a}}$ \\
Chyl. + VLDL cholesterol & $99.7 \pm 6.6^{\mathrm{b}}$ & $76.2 \pm 2.5^{\mathrm{a}}$ & $85.8 \pm 4.7^{\mathrm{ab}}$ \\
LDL cholesterol & $17.1 \pm 2.7^{\mathrm{a}}$ & $13.3 \pm 1.6^{\mathrm{a}}$ & $13.4 \pm 0.9^{\mathrm{a}}$ \\
HDL cholesterol & $40.2 \pm 2.3^{\mathrm{a}}$ & $46.7 \pm 0.4^{\mathrm{b}}$ & $39.3 \pm 2.4^{\mathrm{a}}$ \\
Free cholesterol & $24.8 \pm 1.6^{\mathrm{a}}$ & $22.7 \pm 1.0^{\mathrm{a}}$ & $23.4 \pm 1.7^{\mathrm{a}}$ \\
Triglyceride & $151 \pm 7.7^{\mathrm{a}}$ & $141 \pm 8.9^{\mathrm{a}}$ & $142 \pm 6.0^{\mathrm{a}}$ \\
Phospholipid & $133 \pm 4.5^{\mathrm{a}}$ & $133 \pm 4.1^{\mathrm{a}}$ & $122 \pm 4.5^{\mathrm{a}}$ \\
& \multicolumn{2}{c}{$\mathrm{nmol}$ cholesterol esterified $/ \mathrm{ml} / \mathrm{h}$} \\
LCAT activity & $81.8 \pm 5.9^{\mathrm{a}}$ & $122 \pm 5.4^{\mathrm{c}}$ & $106 \pm 4.4^{\mathrm{b}}$ \\
\hline
\end{tabular}

SP-Oil, safflower oil and palm oil $(8: 2)$; Saf-PL, safflower phospholipid; Soy-PL, soybean phospholipid. * Mean \pm SE $(n=10)$. Means in the same horizontal column not sharing a common superscript letter are significantly different $(p<0.05)$.

Table 4. Concentration of various lipids in liver of rats fed the experimental diets for 4 weeks.

\begin{tabular}{ccccc}
\hline Dietary fat & Total lipid & Total cholesterol & Triglyceride & Phospholipid \\
\hline & & & $\mathrm{mg} / \mathrm{g}$ & \\
SP-Oil & $200 \pm 17.6^{\mathrm{c} *}$ & $48.9 \pm 1.2^{\mathrm{c}}$ & $97.8 \pm 7.1^{\mathrm{c}}$ & $27.8 \pm 1.9^{\mathrm{a}}$ \\
Saf-PL & $93.0 \pm 4.3^{\mathrm{a}}$ & $14.2 \pm 1.1^{\mathrm{a}}$ & $39.0 \pm 2.9^{\mathrm{a}}$ & $36.1 \pm 1.5^{\mathrm{b}}$ \\
Soy-PL & $133 \pm 7.1^{\mathrm{b}}$ & $28.4 \pm 1.6^{\mathrm{b}}$ & $56.2 \pm 4.2^{\mathrm{b}}$ & $33.3 \pm 1.3^{\mathrm{b}}$ \\
\hline
\end{tabular}

SP-Oil, safflower oil and palm oil $(8: 2)$; Saf-PL, safflower phospholipid; Soy-PL, soybean phospholipid. ${ }^{*}$ Mean \pm SE $(n=10)$. Means in the same column not sharing a common superscript letter are significantly different $(p<0.05)$.

SP-Oil diet. The level of chylomicron plus VLDL cholesterol was lower in rats fed the Saf-PL and Soy-PL diets than that of the SP-Oil diet; Saf-PL indicated the lowest value.

\section{LCAT activity}

As shown in Table 3, the LCAT activity was significantly higher when rats were fed the Saf-PL and Soy-PL diets than when fed the SP-Oil diet; Saf-PL indicated the highest value.

\section{Liver lipids}

Liver lipid concentrations of rats fed the various experimental diets are shown in Table 4. The concentrations of liver cholesterol in rats fed the Saf-PL and Soy-PL diets decreased markedly in comparison with those of the SP-Oil diet; Saf-PL indicated the lowest value. Saf-PL and Soy-PL induced a reduction in liver 
Table 5. Excretion of neutral and acidic steroids into feces in rats fed the experimental diets.

\begin{tabular}{cccc}
\hline Dietary fat & SP-Oil & Saf-PL & Soy-PL \\
\hline & & $\mathrm{g} / 2$ days \\
Feces weight & $6.08 \pm 0.25^{\mathrm{a} *}$ & $6.85 \pm 0.13^{\mathrm{b}}$ & $6.78 \pm 0.13^{\mathrm{b}}$ \\
& & $\mathrm{mg} / 2$ days & \\
& $5.71 \pm 1.26^{\mathrm{a}}$ & $47.5 \pm 4.25^{\mathrm{c}}$ & $23.4 \pm 3.36^{\mathrm{b}}$ \\
Total neutral steroids & $0.54 \pm 0.18^{\mathrm{a}}$ & $0.87 \pm 0.13^{\mathrm{a}}$ & $4.02 \pm 1.06^{\mathrm{b}}$ \\
Coprostanol & $5.17 \pm 1.09^{\mathrm{a}}$ & $42.4 \pm 3.77^{\mathrm{c}}$ & $19.4 \pm 3.33^{\mathrm{b}}$ \\
Cholesterol & $89.8 \pm 3.48^{\mathrm{b}}$ & $77.3 \pm 3.00^{\mathrm{a}}$ & $84.3 \pm 2.39^{\mathrm{ab}}$ \\
\hline Total acidic steroids &
\end{tabular}

SP-Oil, safflower oil and palm oil $(8: 2)$; Saf-PL, safflower phospholipid; Soy-PL, soybean phospholipid. *Mean $\pm \mathrm{SE}(n=10)$. Means in the same horizontal column not sharing a common superscript letter are significantly different $(p<0.05)$.

total lipid and triglyceride in comparison with SP-Oil; Saf-PL indicated a remarked reduction. The liver phospholipid was slightly higher with the phospholipid diets than with the triglyceride diet.

\section{Fecal lipids}

Feces dry weight and excretion of neutral and acidic steroids into feces in rats fed the experimental diets are shown in Table 5. Feces weight $(\mathrm{g} / 2$ days) in rats fed the Saf-PL and Soy-PL diets were higher than that in rats fed the SP-Oil diet. Both Saf-PL and Soy-PL caused an enhanced excretion of neutral steroids into feces compared with SP-Oil; Saf-PL indicated the highest value. The increase due to Saf-PL and Soy-PL of fecal neutral steroids was more prominent in cholesterol than in coprostanol. The excretion of coprostanol into feces of rats fed the Soy-PL diets was increased compared with that of other diets. The excretion of acidic steroids into feces did not differ among the various groups.

\section{DISCUSSION}

In a previous study we reported that the safflower phospholipids suppress the elevation of plasma and liver cholesterol in rats fed a hypercholesterolemic diet. We also showed that there is a significant difference of food intake between safflower phospholipid and triglyceride mixture (safflower oil:palm oil=8:2). Therefore, in the present study we used the pair-feeding as the feeding procedure in order to equalize their food intakes. This study was carried out in order to contrast the effect of dietary safflower phospholipid and soybean phospholipid on the cholesterol metabolism in plasma and liver of hypercholesterolemic rats. Both safflower phospholipid and soybean phospholipid were almost the same fatty acid composition, but their phospholipid class compositions were different. In this experiment, we showed that concentrations of plasma cholesterol in rats fed 
safflower phospholipid and soybean phospholipid were reduced. This result was similar to those of the previous experiments in rats $(5-7,9,10)$ and humans $(1)$. In addition to the reduction of plasma cholesterol, liver cholesterol also decreased. Safflower phospholipid indicated a marked reduction in liver total lipid, cholesterol and triglyceride compared with soybean phospholipid. Moreover, the plasmic activity of GOT and GPT were lower in rats fed the phospholipid diets. These results indicated that, in addition to soybean phospholipid, safflower phospholipid may have a significant role in the regulation of lipid metabolism in liver.

The concentrations of total cholesterol in the different lipoprotein fractions resulted in the favorable alteration when safflower phospholipid was fed. The changes in rats fed safflower phospholipid were a reduction in the level of chyromicron plus VLDL cholesterol and a rise in the level of HDL cholesterol. Soybean phospholipid also indicated a reduction in the level of chyromicron plus VLDL cholesterol. The rise of HDL cholesterol was similar to those of the previous experiments in rats $(9,10)$ and humans $(1)$. Imaizumi et al. (7) reported that soybean phospholipid decreased the concentration of serum apolipoprotein A-I (apoA-I). Jimenez et al. (9) indicated that apoA-I disappeared in VLDL, intermediate density lipoprotein (IDL), and LDL after lecithin feeding. However, in this experiment the concentrations of apoA-I in plasma and lipoprotein fraction were not determined. Therefore, we do not know whether safflower phospholipid reduces the concentration of apoA-I in plasma and lipoprotein fractions. These reductions of apoA-I remain to be studied.

In this experiment, it is shown that after safflower phospholipid and soybean phospholipid feeding there is a remarkable increase in LCAT activity; safflower phospholipid indicated the highest value. This result was similar to those of the previous experiments in rats $(9,10)$. Zierenberg and Grundy $(18)$ reported that the absorbed lecithin was incorporated preferentially into the HDL. It has been shown that polyunsaturated lecithin is a better substrate for LCAT (19). We supposed that safflower phospholipid may increase the formation of mature HDL via activation of LCAT in the previous experiment (10). In this experiment, it is shown that safflower phospholipid induces an increase in LCAT activity and a rise in HDL cholesterol level. However, soybean phospholipid induces a slight increase in LCAT activity, but not a rise in HDL cholesterol level. These results suggest that safflower phospholipid may be a better substrate for LCAT than soybean phospholipid.

Both safflower phospholipid and soybean phospholipid increased the excretion of fecal neutral steroids, but not acidic steroids, compared with triglyceride mixture; safflower phospholipid indicated the highest value in fecal neutral steroids. The similar effect of dietary lecithin on the excretion of neutral steroids has been reported in rats fed cholesterol-enriched diet $(20)$ and cholesterol-free diet $(8,21)$ and humans (3). Imaizumi et al. (21) suggested that not only phosphatidylcholine (PC) but also phosphatidylethanolamine (PE) in diet was effective to interrupt the reabsorption of biliary cholesterol in the intestine in rats fed cholesterol-free diet. 
However, Jimenez et al. (9) reported that fecal excretion of neutral sterols was unmodified by polyunsaturated lecithin whereas acidic steroid excretion increased after lecithin feeding in hypercholesterolemic rats. They suggested that fecal bile acids could be derived from an increased catabolism of cholesterol in liver. These different results may be partially explained by the different concentrations of dietary phospholipid used in the respective experiments. In this experiment, we suppose that the increased excretion of fecal neutral steroids may be brought about by inhibiting the absorption of cholesterol in the small intestine.

We showed that safflower phospholipid has the advantage of an increase of the level of HDL cholesterol, a reduction of liver cholesterol and an enhanced excretion of fecal neutral steroids in comparison with soybean phospholipid. However, the mechanisms of the different results between safflower phospholipid and soybean phospholipid cannot be explained by this experiment. Imaizumi et al. (21) demonstrated that $\mathrm{PE}$ is responsible for the alterations of profiles of serum lipids and apoproteins in rats. Ishida et al. (22) suggested that the inositol moiety of phosphatidylinositol (PI) may have a significant role in the regulation of lipid metabolism. Therefore, further work is necessary to evaluate the roles of the respective phospholipids in plasma lipoprotein metabolism.

\section{REFERENCES}

1) Childs, M. T., Bowlin, J. A., Ogilvie, J. T., Hazzard, W. R., and Albers, J. J. (1981): The contrasting effects of a dietary soya lecithin product and corn oil on lipoprotein lipids in normolipidemic and familial hypercholesterolemic subjects. Atherosclerosis, 38, 217-228.

2) Cobb, M., Tukki, P., Linscheer, W., and Raheja, K. (1980): Lecithin supplementation in healthy volunteers. Effect on cholesterol esterification and plasma, and bile lipids. Nutr. Metab., 24, 228-237.

3) Greten, H., Raetzer, H., Stichl, A., and Schettler, G. (1980): The effect of polyunsaturated phosphatidylcholine on plasma lipids and fecal sterol excretion. Atherosclerosis, 36, 81-88.

4) Clark, S. B., Clark, V. E., and Small, D. M. (1981): Effects of lecithin ingestion on plasma and lymph lipoproteins of normo- and hyperlipemic rats. Am. J. Physiol., 241, G422-G430.

5) Samochowiec, L., Kadlubowska, D., and Rozewicka, L. (1976): Investigations in experimental atherosclerosis. Part 1. The effects of phosphatidylcholine (EPL) on experimental atherosclerosis in white rats. Atherosclerosis, 23, 305-317.

6) Kobatake, Y., Kuroda, K., Saito, M., Nishide, E., and Yamaguchi, M. (1988): Differential effect of a dietary soybean phosphatidylcholine and phospholipid mixture on hematologic parameters, and serum lipids in rats. Nippon Eiyo Shokuryo Gakkaishi (J. Jpn. Soc. Nutr. Food Sci. ), 41, 457-463.

7) Imaizumi, K., Murata, M., and Sugano, M. (1982): Effect of dietary polyunsaturated phospholipid on the chemical composition of serum lipoproteins in rat. J. Nutr. Sci. Vitaminol., 28, 281-294. 
8) Murata, M., Imaizumi, K., and Sugano, M. (1982): Effect of dietary phospholipids and their constituent bases on serum lipids and apolipoproteins in rats. J. Nutr., 112, $1805-1808$

9) Jimenez, M. A., Scarino, M. L., Vignolini, F., and Mengheri, E. (1990): Evidence that polyunsaturated lecithin induces a reduction in plasma cholesterol level and favorable changes in lipoprotein composition in hypercholesterolemic rats. J. Nutr., 120, 659667.

10) Iwata, T., Hoshi, S., Tsutsumi, K., Furukawa, Y., and Kimura, S. (1991): Effect of dietary safflower phospholipid on plasma and liver lipids in rats fed a hypercholesterolemic diet. J. Nutr. Sci. Vitaminol., 37, 591-600.

11) Harper, A. E. (1959): Amino acid balance and imbalance. I. Dietary level of protein and amino acid imbalance. J. Nutr., 68, 405-418.

12) Watanabe, M., Itoh, T., Iida, M., Okabe, A., Kawaguchi, Y., Shimbo, K., Sono, R., Murui, T., and Kaneko, T. (1986): Quantitative analysis of phospholipid composition by two dimensional thin-layer chromatography followed by lipid phosphorus determination. Yukagaku (J. Jpn. Oil Chem. Soc.), 35, 1018-1024.

13) Metcalfe, L. D., and Schmitz, A. A. (1961): The rapid preparation of fatty acid esters for gas chromatographic analysis. Anal. Chem., 33, 363-364.

14) Havel, R. J., Eder, H. A., and Bragdon, J. H. (1955): The distribution and chemical composition of ultracentrifugally separated lipoproteins in human serum. J. Clin. Invest., 34, 1345-1353.

15) Folch, J., Lees, M., and Stanley, G. H. S. (1957): A simple method for the isolation and purification of total lipids from animal tissues. J. Biol. Chem., 226, 497-509.

16) Stokke, K. T., and Norum, K. R. (1971): Determination of lecithin : cholesterol acyltransfer in human blood plasma. Scand. J. Clin. Lab. Invest., 27, 21-27.

17) Ide, T., and Horii, M. (1987): A simple method for the extraction and determination of non-conjugated and conjugated luminal bile acids in rats. Agric. Biol. Chem., 51, 3155-3157.

18) Zierenberg, O., and Grundy, S. M. (1982): Intestinal absorption of polyenephosphatidylcholine in man. J. Lipid Res., 23, 1136-1142.

19) Assmann, G. (1976): LCAT lipoprotein and phospholipid substrate specificity, in Phosphatidylcholine, ed. by Peeter, H., Springer-Verlag, Berlin, pp. 34-47.

20) O'Mullane, Y. E., and Hawthorne, J. N. (1982): A comparison of the effects of feeding linoleic acid-rich lecithin or corn oil on cholesterol absorption and metabolism in the rat. Atherosclerosis, 45, 81-90.

21) Imaizumi, K., Mawatari, K., Murata, M., Ikeda, I., and Sugano, M. (1983): The contrasting effect of dietary phosphatidylethanolamine and phosphatidylcholine on serum lipoproteins and liver lipids in rats. J. Nutr., 113, 2403-2411.

22) Ishida, T., Koba, K., Sugano, M., Imaizumi, K., Watanabe, S., and Minoshima, R. (1988): Cholesterol levels and eicosanoid production in rats fed phosphatidylinositol or soybean lecithin. J. Nutr. Sci. Vitaminol., 34, 237-244. 